\title{
Azimuth Phase Center Adaptation on Transmit for High-Resolution Wide-Swath SAR Imaging
}

\author{
Nicolas Gebert and Gerhard Krieger, Member, IEEE
}

\begin{abstract}
Synthetic aperture radar (SAR) systems with multiple receive channels allow for high-resolution wide-swath imaging thus overcoming a fundamental limitation of conventional single-aperture SAR. By using multiple apertures in azimuth, additional samples are received for each transmitted pulse. This allows for a reduced pulse repetition frequency (PRF) thereby enabling a wider swath. However, a nonoptimum PRF is associated with a nonuniform sample spacing in azimuth and needs to be compensated by a multichannel reconstruction algorithm. For strong deviations from the optimum PRF, the inverse character of such an algorithm might result in a degraded performance. This can be overcome by an innovative advanced transmit antenna architecture which allows for a pulse-to-pulse shift of the phase center. Such an antenna enables the adaptive adjustment of the system's phase center positions to the respective PRF, thereby ensuring constant performance over a clearly extended PRF range. In particular, in combination with conventional multichannel processing strategies, this technique represents the next step toward a fully active multiple-input multiple-output (MIMO) SAR and has a great potential for future systems.
\end{abstract}

Index Terms-Digital beamforming (DBF) on receive, highresolution wide-swath synthetic aperture radar (SAR), optimized multichannel azimuth processing, phase center adaptation.

\section{INTRODUCTION}

$\mathbf{F}$ UTURE remote sensing of the Earth requires sensors which provide detailed imagery with continuous global coverage. Due to a system-inherent limitation, synthetic aperture radar (SAR) systems relying only on a single transmit and receive aperture are incapable of such a high-resolution wideswath imaging [1]. This restriction can be overcome by systems with multiple independent receiving channels. For the case of multiple azimuth channels, the principle is shown in Fig. 1: Compared to a single-aperture system (cf., Fig. 1, top), multiple receivers gather for the same pulse repetition frequency (PRF) additional samples, as indicated on the bottom. The sample positions are governed by the spacing $\Delta x$ of the $N$ receivers in combination with the distance between subsequent pulses given by the sensor velocity $v_{\mathrm{s}}$ and PRF. Consequently, a uniform sample distribution is obtained only if the following timing requirement is fulfilled [2]:

$$
\mathrm{PRF}_{\mathrm{uni}}=\frac{2 \cdot v_{\mathrm{s}}}{N \cdot \Delta x}
$$

Manuscript received March 23, 2009. First published August 25, 2009; current version published October 14, 2009.

The authors are with the Microwaves and Radar Institute, German Aerospace Center, 82230 Wessling, Germany (e-mail: nico.gebert@dlr.de; gerhard. krieger@dlr.de).

Color versions of one or more of the figures in this paper are available online at http://ieeexplore.iee.org.

Digital Object Identifier 10.1109/LGRS.2009.2025245

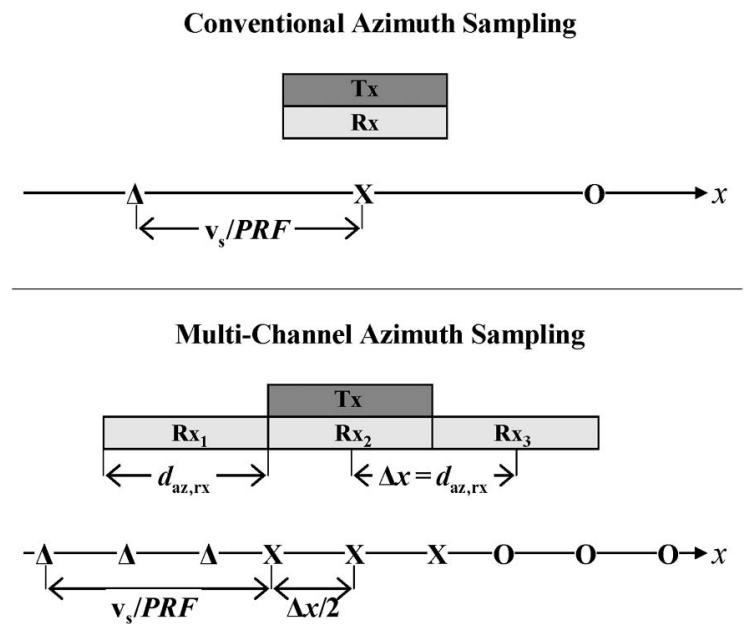

Fig. 1. (Top) Conventional single-aperture SAR and (bottom) multichannel system with corresponding samples in azimuth dimension $x$. For the same PRF, the multichannel system gathers additional samples whose positions are determined by the receiver spacing.

If (1) is violated, additional signal processing is required to cope with a data array resulting from nonequally spaced sample positions in azimuth and avoid degraded performance. Thorough analysis of such a multichannel reconstruction algorithm proved its capability to recover the azimuth signal from nonequally spaced samples independent of the PRF, but revealed for increasing offset from the optimum PRF value a degraded signal-to-noise ratio (SNR), which is caused by the inverse character of the reconstruction filter network [3]. Finally, this restricts the usable PRF range, thus demanding for optimization strategies which increase the system flexibility. As an innovative solution, this letter presents a novel transmit antenna architecture using multiple individually controlled apertures. By this, the effective phase center positions of the multichannel system are adjusted from pulse to pulse in order to match them to the PRF, thus increasing the usable PRF range.

This letter is organized as follows. First, Section II introduces a multichannel system example to image a swath of $100 \mathrm{~km}$ with a geometric resolution in azimuth of $1 \mathrm{~m}$. Furthermore, Section II demonstrates how a conventional digital beamforming (DBF) approach fails to ensure the required performance over the complete PRF range. Based on this result, Section III introduces and analyzes the new idea of adapting the transmit antenna phase center from pulse to pulse in order to compensate for a nonoptimum PRF value. After the theoretical description, Section IV demonstrates the potential of the phase center adaptation technique by means of a system performance example. This letter closes with a discussion containing an outlook on future developments like the extension to fully active 
TABLE I

System PARAmeters And AZIMUth Antenna Dimensions

\begin{tabular}{lcc}
\hline \hline \multicolumn{1}{c}{ Parameter (Azimuth) } & Symbol & Value \\
\hline Carrier wavelength & $\lambda$ & $0.031 \mathrm{~m}$ \\
Coverage (incident angle) & $\Theta_{\mathrm{i}}$ & $20^{\circ}-55^{\circ}$ \\
Geometric resolution & $\delta_{\mathrm{az}}$ & $\leq 1 \mathrm{~m}$ \\
Swath width on ground & $W_{\mathrm{g}}$ & $\geq 100 \mathrm{~km}$ \\
Orbit height & $h_{\mathrm{s}}$ & $580 \mathrm{~km} \pm 10 \mathrm{~km}$ \\
Sensor velocity & $v_{\mathrm{s}}$ & $7560 \mathrm{~m} / \mathrm{s}$ \\
Desired $P R F$ range & $P R F$ & $1150 \mathrm{~Hz}-1550 \mathrm{~Hz}$ \\
Overall Rx antenna length & $l_{\mathrm{az}}$ & $12.25 \mathrm{~m}$ \\
Rx sub-aperture size & $d_{\mathrm{az}, \mathrm{xx}}$ & $1.75 \mathrm{~m}$ \\
Number of "virtual" Rx channels & $N$ & 7 \\
Tx antenna size (effective length) & $d_{\mathrm{az}, \mathrm{tx}}$ & $3.15 \mathrm{~m}$ \\
Processed Doppler bandwidth & $B_{\mathrm{D}}$ & $7.6 \mathrm{kHz}$ \\
\hline \hline
\end{tabular}

multiple-input multiple-output (MIMO) SAR systems with multiple transmit apertures.

\section{REFERENCE SYSTEM}

The present chapter introduces an example system with the parameters summarized in Table I. As the focus of the multichannel processing and the system optimization is on azimuth, only this dimension and the respectively relevant parameters are considered in the following. The system is designed for a PRF range from 1150 to $1550 \mathrm{~Hz}$. According to (1), an overall receive $(\mathrm{Rx})$ antenna length of $12.25 \mathrm{~m}$ is chosen, entailing an optimum PRF of $1236 \mathrm{~Hz}$. Furthermore, $N=7$ receiving channels are necessary to ensure an effective sampling rate high enough for the required resolution, yielding a subaperture length of $1.75 \mathrm{~m}$. The transmit (Tx) antenna length is $3.15 \mathrm{~m}$. A detailed derivation of the system design also including elevation dimension is given in [3].

In the following, the system performance is analyzed, assuming a digital signal processing network according to the multichannel reconstruction algorithm explained in [3]. This algorithm is based on a network of inverse filters, possibly resulting in a degraded SNR if the PRF deviates strongly from the optimum value. As it was shown in [3], the ratio of input to output SNR, normalized to the ratio obtained for uniform sampling, is quantified by $\Phi_{\mathrm{bf}}$, where $P_{j}(f, \mathrm{PRF})$ represents the filter function of channel $j$ and calculation of the mean value is indicated by the operator $\mathbf{E}[\cdot]$

$$
\begin{aligned}
\Phi_{\mathrm{bf}}(\mathrm{PRF}) & =\frac{\left(\mathrm{SNR}_{\mathrm{in}} / \mathrm{SNR}_{\mathrm{out}}\right)}{\left.\left(\mathrm{SNR}_{\mathrm{in}} / \mathrm{SNR}_{\mathrm{out}}\right)\right|_{\mathrm{PRF}_{\mathrm{uni}}}} \\
& =N \cdot \sum_{j=1}^{N} \mathrm{E}\left[\left|P_{j}(f, \mathrm{PRF})\right|^{2}\right] .
\end{aligned}
$$

Fig. 2 shows the simulated results for $\Phi_{\mathrm{bf}}$ considering the full Doppler bandwidth $N$. PRF (solid red line) and a limited bandwidth of $B_{\mathrm{D}}=7.6 \mathrm{kHz}$ (dashed blue line) versus the PRF range. The analytic prediction is given by the diamond symbols, which prove a very good match to the simulations (lines).

$\Phi_{\text {bf }}$ shows sufficiently low values from $1150 \mathrm{~Hz}$ up to $\sim 1370 \mathrm{~Hz}$, but yields unacceptably high values for the PRF range above $1400 \mathrm{~Hz}$ (cf., Fig. 2). The increasing improvement of the curve representing the band-limited image with rising

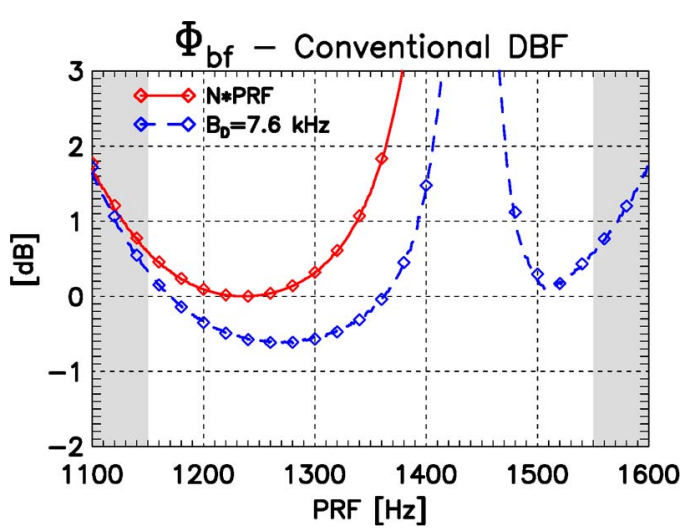

Fig. 2. Simulated SNR scaling factor $\Phi_{\mathrm{bf}}$ of conventional DBF network for (solid red) full and (dashed blue) limited bandwidth $B_{\mathrm{D}}=7.6 \mathrm{kHz}$. Diamond symbols give the analytic prediction.

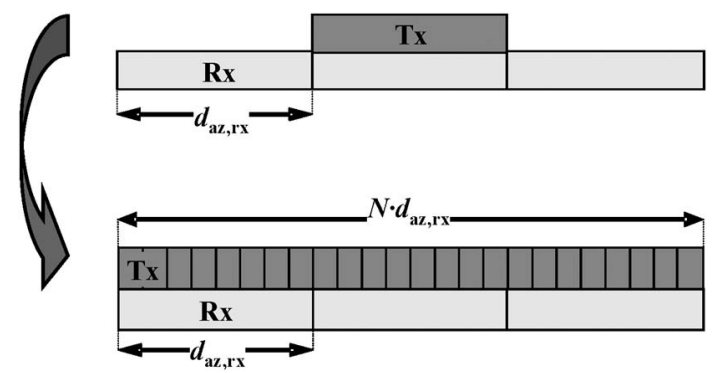

Fig. 3. Novel transmit antenna architecture with increased length and consisting of (bottom) multiple individual elements compared to (top) classical one-element transmit antenna of small size.

PRF results from the increasing oversampling of the signal, as this yields a reduced noise power due to the low-pass filtering inherent to focusing with a fixed Doppler bandwidth. ${ }^{1}$ According to Fig. 2, the conventionally operated multichannel system cannot provide a low SNR scaling - and consequently an acceptable NESZ - over the complete PRF range. This severe restriction can be overcome by the advanced transmit antenna concept that is introduced in the next section.

\section{Phase Center Adaptation on Transmit}

This section presents an advanced transmit antenna architecture in azimuth which allows for the compensation of nonoptimum PRF values by a pulse-to-pulse shift of the transmit phase center. Section III-A introduces the basic principle of the proposed technique, followed by a theoretical analysis in Section III-B. A system example demonstrating the potential of the phase center adaptation is given in Section IV.

\section{A. System Architecture and Basic Principle}

In contrast to a single fixed transmit antenna (Fig. 3, top) the innovative approach is based on a long transmit antenna, which consists of a large number of individually controllable elements (cf., Fig. 3, bottom). In particular, with transmit/receive module technology, this allows for benefiting from the long receive antenna without increasing the antenna dimensions.

\footnotetext{
${ }^{1}$ Note that $\Phi_{b f}$ relates the output to the respective input SNR, but does not take into account a possible variation of the input SNR over the PRF as it is, e.g., encountered for a constant duty cycle.
} 

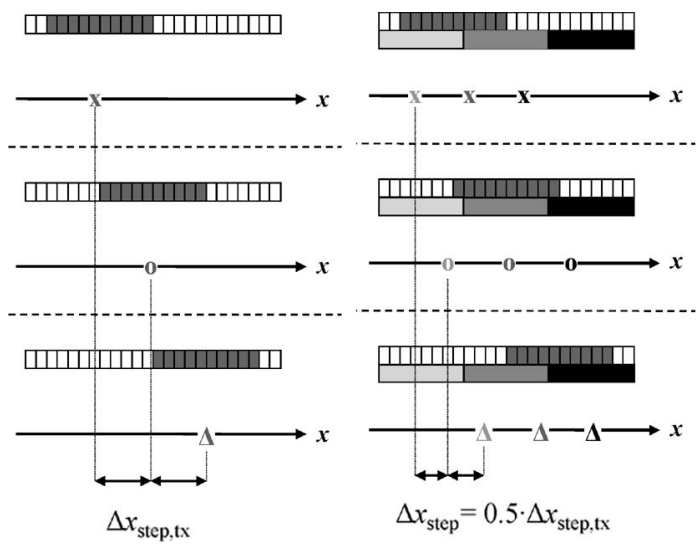

Fig. 4. (Left) Adaptive shift of transmit antenna phase center according to active elements marked in red. (Right) Resulting spatial samples for a multichannel system with varying transmit phase center.

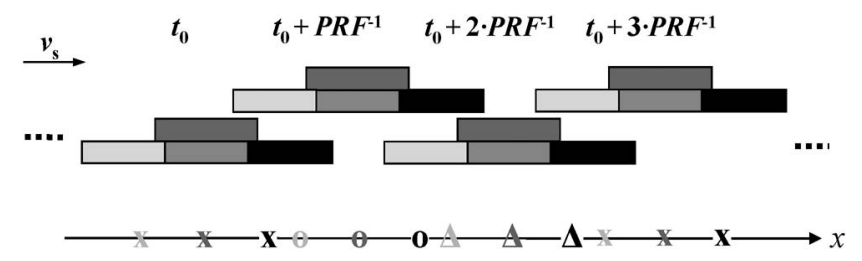

Fig. 5. Multichannel system with conventional transmit antenna operated at nonoptimum PRF entailing nonuniformly spaced samples, exemplarily given for four subsequent pulses.

Such an antenna permits to change the size of the effective Tx aperture and its position on the antenna by activating the respective elements. This allows for adaptively varying the transmit phase center position by using only specific parts of the antenna. Activating different antenna elements from pulse to pulse yields a phase center in azimuth that "travels" over the antenna as shown exemplarily for three subsequent pulses in Fig. 4 on the left. Taking further into account the receiver phase centers of a conventional multichannel antenna, the resulting effective phase centers of the system can be shifted by half of the displacement of the transmit antenna phase center with respect to its center position. A system with, for example, three receive apertures is shown in Fig. 4 on the right.

This capability to adjust the spatial samples from pulse to pulse allows for compensating nonoptimum PRF values: According to the actual PRF, the step size of the phase center variation from pulse to pulse is set flexibly thus yielding a uniform -or at least a less nonuniform—sample spacing. A simple example to illustrate the basic principle is given in the following: Fig. 5 shows a conventional multichannel SAR system operated with a nonoptimum PRF. The azimuth positions of the nonuniformly distributed samples received for four transmit pulses are shown at the bottom where the samples arising from different pulses are coded with different symbols while all samples corresponding to the same receive subaperture are marked by the same shade of gray.

Fig. 6 shows how an adaptive shift of the transmit aperture from pulse to pulse can compensate for the nonoptimum PRF yielding uniform sampling. In this exemplary case, the cycle time after which the phase center has reached its outermost position and "jumps" back to the opposite antenna end is chosen to three pulses. Hence, as the nonoptimum PRF is assumed to be too high, every fourth pulse two samples coincide and have

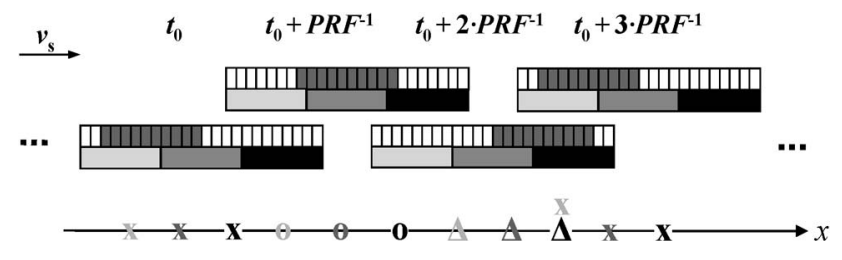

Fig. 6. Multichannel system with pulse-to-pulse phase center adaptation on transmit that compensates for the nonoptimum PRF and finally yields uniformly spaced samples.

to be averaged or skipped. Note that Fig. 6 shows a special case, where the transmit phase center starts the new cycle at its original starting position.

\section{B. Analytic Description}

As a next step, the present section derives an analytic description of the technique. First, (3) quantifies the necessary shift $\Delta x_{\text {step }}$ of the samples that is needed from pulse to pulse to compensate for the nonoptimum PRF if the optimum value is given by $\mathrm{PRF}_{\mathrm{uni}}=2 \cdot v_{\mathrm{s}} / l_{\mathrm{az}}$, with the sensor velocity $v_{\mathrm{s}}$ and the receive antenna length in azimuth $l_{\mathrm{az}}$

$$
\Delta x_{\text {step }}=\frac{v_{\mathrm{s}}}{\mathrm{PRF}_{\mathrm{uni}}}-\frac{v_{\mathrm{s}}}{\mathrm{PRF}}=\frac{l_{\mathrm{az}}}{2}-\frac{v_{\mathrm{s}}}{\mathrm{PRF}} .
$$

Taking into account the effective phase centers midway between transmitter and receiver, the necessary shift of the transmit aperture per pulse is given by $\Delta x_{\text {step }, \text { tx }}=2 \cdot \Delta x_{\text {step }}$ to ensure the necessary shift of the phase centers. To calculate the phase center position from pulse to pulse, the initial position of the transmit phase center is assumed at its outermost position $x_{\mathrm{tx}, \max }$ that is defined by (4), where $d_{\mathrm{az}, \mathrm{tx}}$ represents the length of the activated transmit aperture in azimuth. The " \pm " specifies the maximum fore or aft position, respectively, with respect to the flight direction

$$
x_{\mathrm{tx}, \max }= \pm\left(\frac{l_{\mathrm{az}}}{2}-\frac{d_{\mathrm{az}, \mathrm{tx}}}{2}\right) .
$$

Then, the phase center position $x_{\mathrm{tx}}(t)$ is shifted by $\Delta x_{\mathrm{step}, \mathrm{tx}}$ between subsequent pulses according to (5) as long as the opposite maximum position on the antenna is not reached, i.e., (6) has to be fulfilled

$$
\begin{aligned}
x_{\mathrm{tx}}\left(t+\mathrm{PRF}^{-1}\right) & =x_{\mathrm{tx}}(t)+\Delta x_{\text {step }, \mathrm{tx}} \\
\mp x_{\mathrm{tx}, \max } \pm k \cdot \Delta x_{\text {step }, \mathrm{tx}} & \leq \pm x_{\mathrm{tx}, \max } ; k \in \mathbb{N} .
\end{aligned}
$$

When (6) does not hold true any more, the phase center shifts backward or forward, toward the opposite side of the antenna. Performing this shift, it has to be assured that the position of two subsequent transmit phase centers yields again an effective phase center distance that corresponds to the uniform PRF. Consequently, the distance of the transmit phase center has to include the necessary shift $\Delta x_{\text {step,tx }}$ and an integer multiple of the receive phase center distance $\Delta x$. Assuming a transmit antenna phase center $x_{\mathrm{tx}}\left(t_{0}\right)$ at time $t_{0}$, and a position $x_{\mathrm{tx}}\left(t_{1}\right)$ at the next pulse at time $t_{1}=t_{0}+\mathrm{PRF}^{-1}$, one obtains

$$
\begin{aligned}
x_{\mathrm{tx}}\left(t_{1}\right)-x_{\mathrm{tx}}\left(t_{0}\right) & =\Delta x_{\text {step }, \mathrm{tx}}-q \cdot \Delta x \\
& =m \cdot \Delta x-\frac{2 \cdot v_{\mathrm{s}}}{\mathrm{PRF}}, \quad q, m \in \mathbb{Z} .
\end{aligned}
$$


As mentioned, a shift of the Tx phase center back to the opposite side of the antenna is necessary every time it has reached its outermost position. When this happens, PRF values higher than $\mathrm{PRF}_{\text {uni }}$ entail coinciding spatial sample positions leading to "redundant" samples. In this case, two samples have to be "merged" to a single sample, e.g., by averaging or simply discarding one of them. This yields a uniformly sampled signal independent from the PRF at the "cost" of a ratio of $1-\left(\mathrm{PRF} / \mathrm{PRF}_{\mathrm{uni}}\right)$ redundant samples. If the PRF is below the optimum value, this results in "missing" samples within the synthetic aperture, as a gap occurs in the spatial sampling every time the phase center is switched. Hence, to obtain a uniformly sampled data array, this case requires as an additional step to fill the gaps, e.g., by interpolation techniques. Finally, before focusing the signal with a filter matched to the uniform PRF, the impact on the received multiaperture SAR signal in azimuth has to be considered. The multiaperture impulse response in azimuth derived in [2] and [3] showed a constant phase term, which was dependent on the distance between transmitter and respective receiver. As the transmitter phase center now varies from pulse to pulse, its distance to the receivers changes continuously. This means that depending on the pulse and the respective position of the transmit phase center, a phase $\gamma_{j}$ has to be applied to each of the channels $j$, where $\Delta x_{j}$ is the position of receiver aperture $j, \lambda$ is the wavelength and $R_{0}$ the slant range

$$
\gamma_{j}(t)=\frac{\pi \cdot\left(\Delta x_{j}-x_{\mathrm{tx}}(t)\right)^{2}}{2 \cdot \lambda \cdot R_{0}} .
$$

In a real system, the transmit phase center cannot be adjusted arbitrarily, but is bound to discrete positions that result from the size of the individually controllable antenna elements. Assuming an antenna divided into a number of $K$ elements, the required optimum pulse-to-pulse shift of the transmit phase center by $p$ elements can be calculated from (9), which includes rounding to ensure $p$ to be an integer number

$$
p=\frac{K}{l_{\mathrm{az}}} \cdot \Delta x_{\mathrm{step}, \mathrm{tx}}, \quad p \in \mathbb{Z} .
$$

Furthermore, the fine-tuning ability of the position, i.e., the minimum possible step size of the phase center from pulse to pulse, depends also on the distance between single transmit elements and is given by $l_{\mathrm{az}} / K$. Furthermore, the sign of $p$-and consequently $\Delta x_{\text {step,tx }}$ - defines the direction of the movement of the sliding phase center, either along (for positive values) or against the flight direction (for negative values), to adjust the spatial sampling resulting from too high or too low PRF values, respectively. Rearranging (9), one obtains (10) that can be understood as a generalization of the timing requirement for uniform sampling by replacing the constant overall antenna length $l_{\mathrm{az}}$ by an effective overall length $l_{\mathrm{az}, \text { eff }}$ that can be adapted by the settings for the pulse-to-pulse adaptation

$$
\begin{aligned}
\mathrm{PRF} & =\frac{2 \cdot v_{\mathrm{s}}}{l_{\mathrm{az}}-\Delta x_{\mathrm{step}, \mathrm{tx}}}=\frac{2 \cdot v_{\mathrm{s}}}{l_{\mathrm{az}, \mathrm{eff}}} \\
& =\frac{2 \cdot v_{\mathrm{s}}}{l_{\mathrm{az}}(1-p / K)}=\frac{\mathrm{PRF}_{\mathrm{uni}}}{(1-p / K)} .
\end{aligned}
$$

The PRF range that can be compensated is determined by the maximum possible displacement of the phase center. It is defined by the separation of the outermost positions according to (4). Assuming a transmit aperture size $d_{\mathrm{az}, \mathrm{tx}}$ equal to a single receiver $d_{\mathrm{az}, \mathrm{rx}},(11)$ gives the maximum step size $\Delta x_{\mathrm{step}, \mathrm{tx}, \max }$

$$
\Delta x_{\mathrm{step}, \mathrm{tx}, \max }= \pm\left(l_{\mathrm{az}}-d_{\mathrm{az}, \mathrm{tx}}\right)= \pm(N-1) \cdot d_{\mathrm{az}, \mathrm{rx}} .
$$

Equation (11) defines, dependent on the direction of the step according to its sign, the maximum and minimum possible PRF to be compensated in (12) and (13), respectively

$$
\begin{aligned}
& \mathrm{PRF}_{\text {max }}=\frac{1}{\left(1-\frac{N-1}{N}\right)} \cdot \mathrm{PRF}_{\text {uni }}=N \cdot \mathrm{PRF}_{\text {uni }} \\
& \mathrm{PRF}_{\text {min }}=\frac{1}{\left(1+\frac{N-1}{N}\right)} \cdot \mathrm{PRF}_{\text {uni }}=\frac{1}{\left(2-\frac{1}{N}\right)} \cdot \mathrm{PRF}_{\text {uni }} .
\end{aligned}
$$

Despite the theoretical possibility to compensate PRF values up to $N \cdot \mathrm{PRF}_{\text {uni }}$ (cf., (12)), it should be noted that for $N \geq 3$, additional strategies are possible, as, e.g., uniform sampling of higher order, i.e., a spatial interleaving of temporally succeeding pulses, could be achieved. This means that, in this case, the presented technique would be applied up to a maximum $\mathrm{PRF}$ of $2 \cdot \mathrm{PRF}_{\text {uni }}$. Above this value, the compensation could be adapted to a uniform PRF of higher order, i.e., the pulse-topulse steps are derived for the closest uniform PRF that is below the given PRF.

For the above limits of $\mathrm{PRF}_{\min }$ and $\mathrm{PRF}_{\max }$, this results in coinciding samples every second pulse if the maximum PRF is corrected for, while a gap in the spatial sampling occurs every two pulses if the minimum PRF shall be compensated. Hence, (13) represents only the theoretical value which can be compensated, but does not give information on how frequently gaps in the sampling can be tolerated.

\section{Performance Analysis}

In order to give an example of the benefits arising from this technique, the system presented in Section II is extended by an advanced transmit antenna architecture as shown in Fig. 3 . For reasons of demonstrating the general potential of this technique, the possibility for arbitrarily setting the transmit antenna phase center is assumed. In the presented case, the gaps in the sampling grid for PRF values smaller than $\mathrm{PRF}_{\text {uni }}$ were filled by an interpolation method that closely approximates a sincinterpolation. The resulting azimuth ambiguity-to-signal ratio $\left(A A S R_{N}\right)$ is shown in Fig. 7, where the red line represents the conventional approach, while the blue line is obtained for the pulse-to-pulse adaptation of the Tx phase center.

For PRF values below $\mathrm{PRF}_{\text {uni }}$, the $A A S R_{N}$ slightly increases with respect to the conventional reference, caused by the missing samples at times when the transmit phase center switches. This problem can possibly be mitigated by special preprocessing - e.g., advanced interpolation techniques- to fill the gaps in the spatial sampling, but the investigation of such techniques shall not be subject of this letter. For a PRF higher than the optimum value, the $A A S R_{N}$ stays constant at the value corresponding to the optimum PRF, as in this case, the signal is identical to the uniformly sampled signal (cf., Fig. 7). Compared to the conventional DBF case, the ambiguity suppression is improved up to $2 \mathrm{~dB}$ for a PRF value of $1550 \mathrm{~Hz}$. Regarding the SNR, the big advantage of the adaptive phase 


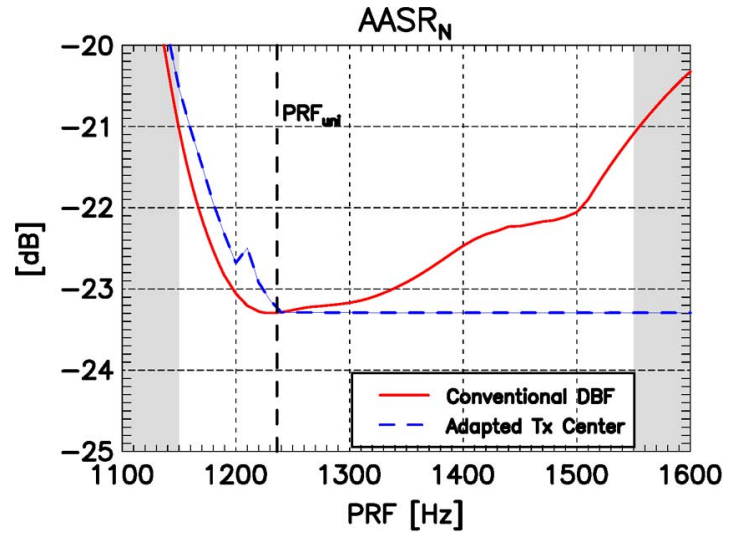

Fig. 7. Ambiguous energy suppression, $A A S R_{N}$, versus PRF for (blue) adaptive phase center shift on transmit compared to (red) conventional DBF.

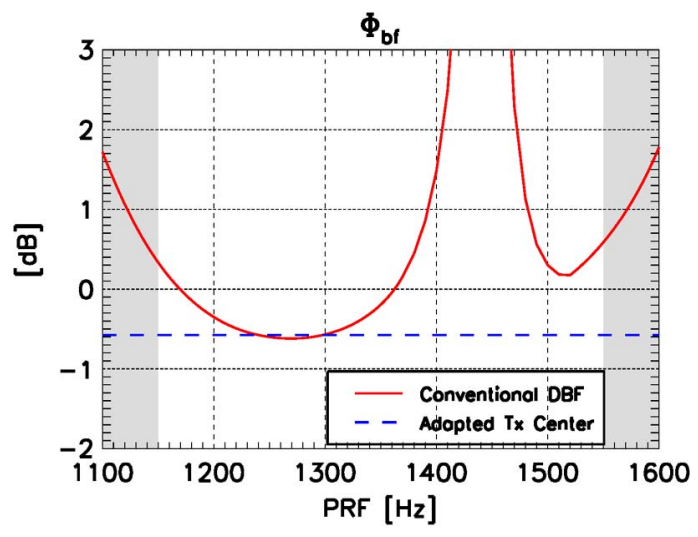

Fig. 8. SNR scaling factor $\Phi_{\mathrm{bf}}$ for (solid red) conventional DBF approach and for (dashed blue) adaptive phase center shift on transmit. $B_{\mathrm{D}}=7.6 \mathrm{kHz}$.

center technique becomes clear. As uniform sampling is ensured for any PRF, no degradation of the SNR is induced by the processing. This means that $\Phi_{\mathrm{bf}}$ remains constant independently of the PRF, and it is defined by the value obtained for uniform sampling (cf., Fig. 8, dashed blue line). Compared to the SNR scaling of the conventional system (solid red), this results in a clear improvement, particularly for high PRF values. $^{2}$

In consequence, the formerly restricted PRF range of operation is extended when applying this technique, thus enabling the operation of the system over the complete required range. Furthermore, the sampling rate does not increase with the PRF as only the number of spatially coinciding pulses increases. On the one hand, this means that even for higher PRF values, the data volume does not increase. On the other hand, one does not benefit from an increasing effective sampling rate with respect to the NESZ, as the effective sampling rate is always determined by the optimum. As a result, fewer samples are received, finally leading to a reduced signal power. In the worst case, given by the ratio of the maximum PRF of $1550 \mathrm{~Hz}$ to the optimum one of $1236 \mathrm{~Hz}$, this yields a "loss" of $\sim 1 \mathrm{~dB}$ according to $\sim 20 \%$ redundant pulses. In this context, it should be noted that

\footnotetext{
${ }^{2}$ A "real" antenna not allowing for arbitrary but only for discrete transmitter positions will result in an improved sampling but show remaining nonuniformity. As a result, $A A S R_{\mathrm{N}}$ and $\Phi_{\mathrm{bf}}$ would no longer be constant but vary according to the respective residual nonuniformity. Nevertheless, the performance would remain better than without compensation.
}

this does not take into account the possibly increased signal power of samples obtained by the combination of two spatially coinciding samples.

Regarding the geometric resolution in azimuth, both techniques provide a constant resolution of $\sim 1 \mathrm{~m}$ above the optimum PRF, while below this value the phase center adaptation entails a moderate degradation of the resolution that increases with increasing offset from the optimum PRF value.

\section{Discussion}

The multichannel reconstruction algorithm represents a promising solution to cope with the challenges posed by multichannel SAR data. Nevertheless, the inverse character of the applied processing network might result in a degraded SNR, particularly if the PRF values deviate strongly from the optimum PRF. In consequence, the applicable PRF range is restricted and optimization techniques become necessary to ensure the required flexibility.

This letter introduced and analyzed such an optimization strategy based on an advanced transmit antenna concept. The antenna architecture consists of multiple individual Tx elements in azimuth that allow for a pulse-to-pulse shift of the transmit antenna phase center such that the spatial distribution of the gathered multichannel samples is adaptively matched to the PRF. In particular, for PRF $>\mathrm{PRF}_{\mathrm{uni}}$, this allows for compensating or at least mitigating the performance loss from a nonuniform sampling of the azimuth signal. Consequently, the proposed method prevents a severe SNR degradation by the processing network and enables to overcome the restricted PRF range, thus leading to a much more flexible system design. Particularly, the combination of the adaptive pulse-to-pulse shift with the "conventional" multichannel reconstruction offers a clearly extended full performance PRF region: The phase center adaptation is used as rather "rough" precorrection stage preceding the fine-correction which is accomplished by the reconstruction network.

In conclusion, the presented advanced antenna architecture can be seen as a further step toward sophisticated transmit antennas, leading in the future to a fully active MIMO SAR. This will enable new concepts such as digital waveform encoding on transmit offering various and powerful opportunities to push the system performance even further [4]-[6].

\section{REFERENCES}

[1] J. Curlander and R. McDonough, Synthetic Aperture Radar-Systems and Signal Processing. Hoboken, NJ: Wiley, 1991.

[2] G. Krieger, N. Gebert, and A. Moreira, "Unambiguous SAR signal reconstruction from nonuniform displaced phase center sampling," IEEE Geosci. Remote Sens. Lett., vol. 1, no. 4, pp. 260-264, Oct. 2004.

[3] N. Gebert, G. Krieger, and A. Moreira, "Digital beamforming on receive: Techniques and optimization strategies for high-resolution wide-swath SAR imaging," IEEE Trans. Aerosp. Electron. Syst., vol. 45, no. 2, pp. 564 592, Apr. 2009.

[4] G. Krieger, N. Gebert, and A. Moreira, "Hochauflösendes SynthetikApertur-Seitensicht-Radarsystem mittels Digital Beamforming," German Patent No. DE 102006022 814.6 / European Patent No. EP 2007 / 003921 , Nov. 15, 2007.

[5] G. Krieger, N. Gebert, and A. Moreira, "Multidimensional waveform encoding: A new digital beamforming technique for synthetic aperture radar remote sensing," IEEE Trans. Geosci. Remote Sens., vol. 46, no. 1, pp. 3146, Jan. 2008.

[6] G. Krieger, N. Gebert, M. Younis, and A. Moreira, "Advanced synthetic aperture radar based on digital beamforming and waveform diversity," in Proc. IEEE RadarCon, Rome, Italy, May 2008, pp. 1-6. 